\title{
Monitoramento e Disseminação Cooperativa de Eventos Emergenciais Apoiado por Agrupamentos de Veículos
}

\author{
Everaldo Andrade ${ }^{1}$, Kevin Veloso ${ }^{1}$, Nathália $\operatorname{Vasconcelos}^{1}$, Aldri Santos ${ }^{2}$, Fernando Matos ${ }^{1}$ \\ ${ }^{1}$ Centro de Informática - Universidade Federal da Paraíba (UFPB) \\ ${ }^{2}$ Departamento de Informática - Universidade Federal do Paraná (UFPR) \\ aldrieinf.ufpr.br, nathalia.vasconcelosdeng.ci.ufpb.br, \\ kevinveloso@gmail.com, everaldo.andrade@ppgi.ci.ufpb.br, fernando@ci.ufpb.br
}

\begin{abstract}
Critical urban events occur at a random way and they need to be dealt with by public authorities quickly to maintain the proper operation of cities. The main challenges for an efficient handling of an event fall precisely in its random nature, and in the speed and accuracy of the notification of its manifestation to the authority. Moreover, the fact that this notification depends on human intervention aggravates this situation. The omnipresence of vehicles in urban environments, and their communication and monitoring capabilities, allow to employ VANETs to support the dissemination and handling of such events. Thus, this paper proposes MINUET, a system to support the monitoring and dissemination of urban events, which operates in an ad hoc vehicular network. It uses a cooperative-based strategy where vehicles are able to dynamically coordinate the monitoring and dissemination of multiple events in real time by the means of clusters. Results obtained by NS3 show the performance of MINUET regarding the real-time monitoring and dissemination of events detected in urban areas.
\end{abstract}

Resumo. Eventos urbanos emergenciais ocorrem de forma aleatória e precisam ser tratados por entidades competentes para manter o bom funcionamento das cidades. Os principais desafios para um tratamento eficiente recaem justamente na aleatoriedade no tempo ou no espaço, e na rapidez e acurácia da comunicação da ocorrência do evento a uma entidade responsável, sendo este último desafio agravado pelo fato desta comunicação depender de intervenção humana. A onipresença de veículos em ambientes urbanos, sua capacidade de comunicação e monitoramento, possibilitam o uso de VANETs como meio de auxílio para disseminação e tratamento destes eventos. Assim, este artigo propõe MINUET, um sistema de monitoramento e disseminação de eventos urbanos, que atua em uma rede veicular não estruturada. Ele é baseado em uma estratégia cooperativa, onde veículos coordenam dinamicamente o monitoramento e a disseminação de múltiplos eventos em tempo real através de agrupamentos. Resultados no NS3 mostram o desempenho do MINUET na detecção, monitoramento e disseminação em tempo real de eventos em zonas urbanas.

\section{Introdução}

Nos últimos anos, os centros urbanos têm vivenciado um crescimento populacional sem precedentes, o qual impactará em vários aspectos do dia-a-dia da cidade, como por 
exemplo na mobilidade urbana. Este aumento populacional dificultará cada vez mais a gestão da cidade por parte das entidades públicas ao agravar aspectos como a mobilidade urbana [Monreal 2018]. Dentre os vários problemas enfrentados pelos gestores das cidades, é possível destacar o tratamento de eventos emergenciais urbanos através da detecção, análise e monitoramento dos eventos [Memos et al. 2018]. Tais eventos podem ser incêndios, acidentes, assaltos, fuga de veículos, pessoas ou veículos procurados, bloqueios, manifestações, dentre vários outros. O tratamento rápido e eficiente destes eventos, através da colaboração entre agentes distribuídos no ambiente, torna-se primordial para garantir a segurança e a assistência dos indivíduos direta ou indiretamente envolvidos [Monajemi et al. 2018]. Além disso, uma resposta rápida garante um bom andamento do dia-a-dia da cidade. Por exemplo, ao detectar um bloqueio em uma avenida, a entidade pública de mobilidade deve agir rapidamente para desobstruir a via de modo a não criar longos e demorados congestionamentos em outras partes da cidade.

A infraestrutura de Tecnologias de Informação e Comunicação (TICs), existente em ambientes de Cidades Inteligentes, pode desempenhar um papel importante no tratamento destes eventos urbanos, onde a intercomunicação entre a infraestrutura urbana e as autoridades pode auxiliar no tratamento de tais eventos. Além disso, com a evolução de áreas como Crowdsourcing [Ma et al. 2018] e IoT [Hassan and Awad 2018], a integração e a cooperação de diferentes sistemas computacionais podem proporcionar o surgimento de novas estratégias no gerenciamento à respostas de emergências e monitoramento diário em zonas urbanas. Desta forma, é possível idealizar a criação de infraestruturas resilientes que garantam a continuidade das funcionalidades das cidades até mesmo em face de eventos catastróficos [Timashev 2017, Furutai and Kanno 2018]. Estas estratégias, normalmente fazem uso de fontes de captadores de informações (e.g. câmeras, sensores, smartphones e veículos) com o objetivo de auxiliar na tomada de decisões de outros sistemas responsáveis pelo tratamento de eventos específicos.

Os Sistemas de Transporte Inteligente (ITS) contribuem para a automatização de operações de deslocamentos dentro das Cidades Inteligentes. Em ITSs, veículos fazem parte de uma Rede Veicular não Estruturada (Vehicular Ad Hoc Network - VANET), através da qual se intercomunicam com outros veículos (Vehicle-to-Vehicle - V2V) e com a infraestrutura de redes da cidade (Vehicle-to-Infrastrucutre - V2I) para trocarem informações para os mais variados objetivos relacionados a segurança e entretenimento. Além disso, devido à sua onipresença, os veículos aparecem como uma alternativa óbvia para a coleta de dados multimídia nos centros urbanos [Wang 2018, Quadros et al. 2015b, Quadros et al. 2015a, Quadros et al. 2016] referente a eventos e para suportar cooperação e coordenação no monitoramento [Castro et al. 2017], uma vez que os mesmos não sofrem da limitação de recursos de processamento, armazenamento e energia que outras redes não estruturadas possuem [Khakpour et al. 2017]. Contudo, as VANETs impõem seus próprios desafios, tais como alta mobilidade [Silva et al. 2016], variação da densidade de tráfego, alta escalabilidade e variação topológica da rede [Khakpour et al. 2013, Macedo et al. 2014].

Embora estudos recentes têm mostrado estratégias ao gerenciamento de eventos em cidades, eles geralmente limitam-se ao tratamento de um evento específico, como por exemplo, inundações [Mousa et al. 2016], acidentes veiculares [Chou et al. 2017] ou condições de tráfego [Abhishek et al. 2016]. Outros trabalhos focam na disseminação de 
conteúdos de vigilância [Huang et al. 2017] ou de colisões [Shi et al. 2017], porém em uma abordagem centralizada para a comunicação do evento. Contudo, a própria infraestrutura em cidades inteligentes poderia permitir uma colaboração através de crowdsourcing de veículos na detecção por imagens e disseminação de eventos emergenciais até uma entidade de controle pública externa com capacidade de processamento para reconhecer eventos e tomar as devidas providências de modo a otimizar as ações públicas.

Desta forma, este trabalho apresenta o modelo de um sistema baseado em agrupamentos cooperativos de veículos chamado MINUET (MonitorINg and Dissemination of Urban EvenTs), que opera sobre uma rede veicular híbrida para suportar a coordenação e o controle do monitoramento distribuído e dinâmico e disseminação sobre múltiplos eventos urbanos (e.g. acidentes, desastres e flagrantes) de modo a auxiliar as autoridades no reconhecimento destes eventos. No modelo proposto, ao se detectar um evento, os veículos se auto-coordenam para monitorar este evento o maior tempo possível de forma à entidade pública tomar a decisão mais eficaz para a sua resolução. Além disso, os veículos também coordenam uma comunicação cooperativa, onde múltiplos veículos transmitem dados de uma única fonte evento, e há a combinação dos dados transmitidos pelos agrupamentos de veículos na entidade destino (entidade competente). O MINUET emprega uma gerência de coordenação e controle adaptativos e distribuídos baseada no contexto temporal e espacial dos veículos (crowdsourcing), onde eles colaborativamente executam o monitoramento e a disseminação de maneira dinâmica e sob-demanda. Os principais diferenciais do MINUET são: (i) o controle distribuído, onde todos os dispositivos com capacidade de entregar as informações, o fazem; diferente dos atuais trabalhos, apenas o líder do grupo decide quais informações são enviadas a uma central de controle ou apenas o líder se comunica com a central; (ii) e o monitoramento colaborativo; diferente de trabalhos, onde apenas o líder monitora o evento, não havendo assim um monitoramento colaborativo. O MINUET foi avaliado através de simulações, e empregou-se o agrupamento de [Tal et al. 2016] para mensurar a eficácia do MINUET no gerenciamento da disseminação de eventos emergenciais. Os resultados mostram que o MINUET consegue detectar um evento cerca de seis vezes por minuto, monitorar e disseminar cerca de $40 \%$ do dados, através de agrupamentos, apoiando assim o tratamento destes eventos.

O restante do artigo está estruturado como descrito a seguir. A Seção 2 apresenta os trabalhos relacionados. A Seção 3 descreve o sistema MINUET e o seu funcionamento. A Seção 4 detalha a metodologia de avaliação aplicada no MINUET e os resultados alcançados. Finalmente, a Seção 5 apresenta as considerações finais do trabalho.

\section{Trabalhos Relacionados}

A onipresença de veículos nas cidades foi uma das causas que impulsionou o desenvolvimento de trabalhos em VANETs. Existem várias soluções na literatura que utilizam VANETs para auxiliar no cotidiano das cidades ao tratar algum aspecto urbano específico. Dentre os vários serviços para cidades inteligentes, alguns utilizam estrutura de agrupamentos para apoiar o rastreamento de objetos-alvos e/ou a disseminação de informações.

Em [Huang et al. 2017] foi proposto um esquema de controle que utiliza algoritmo de agrupamento baseado em área de visão juntamente com técnicas adaptativas de streams de vídeo para auxiliar na vigilância das vias de trânsito. Grupos de veículos propagam informações visuais em tempo real. A solução busca escolher o líder de grupo 
mais apropriado, o qual é o responsável por capturar imagens da via e repassar aos demais membros. Entretanto, apenas o líder do grupo captura as informações, não permitindo assim uma colaboração durante o monitoramento. Os autores em [Shi et al. 2017] propõem uma solução de agrupamento centralizada baseada em uma arquitetura de rede híbrida. Ao usar interfaces IEEE 802.11p e LTE, os veículos podem escolher o modo de comunicação e disseminação de mensagens de alerta para outros grupos da rede através de uma entidade central. Tais mensagens visam alertar os motoristas sobre colisões em cruzamentos e engarrafamentos. Contudo, nesta proposta apenas o líder do grupo se comunica com a rede infraestruturada, o que pode causar atrasos na disseminação das informações.

Em [Wang et al. 2018] os autores propõem um framework para disseminação de conteúdo em redes de acesso heterogêneas usando uma estratégia de gerenciamento de tráfego baseada em agrupamentos. O trabalho utiliza um sistema baseado em crowdsensing para coletar informações de eventos de forma colaborativa de forma a entregá-las a uma central de gerenciamento. Membros do grupo enviam mensagens referentes a um evento para o líder do grupo, onde este analisa os dados de forma a ter informações mais precisas sobre os eventos e decide pela transmissão imediata ou adiada para a central. Em [Khakpour et al. 2017] os autores apresentam e realizam testes em dois algoritmos de agrupamento para rastreamento de veículos em vias de trânsito. Na estratégia aplicada pelos algoritmos, os grupos são formados apenas pelos veículos que atualmente detectam o veículo alvo e por aqueles veículos que possuem uma alta probabilidade de detectar o veículo alvo logo em seguida. O líder do grupo concentra os dados coletados de todos os membros do grupo, analisa os dados de forma a eliminar redundâncias e os retransmite à rede infra-estruturada. Apesar da eficácia, estas duas soluções concentram no líder do grupo a responsabilidade de decidir o que enviar para uma central de controle.

No trabalho proposto em [Yedder and Benyahia 2017], os autores propõem um sistema reativo de disseminação de informação para auxiliar no despacho de veículos de emergência (e.g. uma ambulância). A partir de informações coletadas pelos veículos na VANETs, é possível inferir que uma via está congestionada, o que possibilita que o rota do veículo de emergência possa ser alterada em tempo real. Contudo, são feitas simulações apenas considerando questões de mobilidade e de tráfego, sem levar em conta questões da infraestrutura de rede (e.g. atraso, colisões) poderiam influenciar na solução. Em [Gorrieri et al. 2016] é proposta uma estratégia descentralizada para aquisição de dados de fenômenos em redes veiculares. Numa primeira fase (downlink phase), um nó sorvedouro remoto (pode estar na nuvem) dispara mensagens na rede para formação de grupos. Durante a formação, os líderes de cada grupo também já são definidos. Em uma segunda fase (uplink phase), dados coletados pelos veículos de cada grupo, referentes ao fenômeno observado, são enviados para os seus respectivos líderes e repassados para o nó sorvedouro. A ideia é criar uma topologia baseada em grupos para a disseminação de dados dos fenômenos. Contudo, esta solução emprega uma estratégia pró-ativa, o que pode desperdiçar recursos em casos onde não ocorrem fenômenos.

\section{Sistema de Deteç̧ão e Disseminação de Eventos Emergenciais Urbanos}

Esta seção apresenta o sistema MINUET para o apoio ao monitoramento cooperativo de eventos emergenciais urbanos através de agrupamentos sob demanda de veículos, que emprega uma gerência de coordenação e controle adaptativos e distribuídos baseada no contexto temporal e espacial dos veículos (crowdsourcing). Ele opera sobre VANETs 
híbridas para apoiar o monitoramento de eventos emergenciais e a disseminação dos dados coletados para uma entidade externa que trata os eventos urbanos a partir do seu reconhecimento. Na entidade, os dados são analisados por aplicações alvo de modo a auxiliá-la na tomada de decisões à resolução dos eventos. O MINUET utiliza um controle distribuído, onde os próprios veículos decidem quem irá monitorar e/ou disseminar as informações. Inicialmente, descreve-se o modelo de ambiente urbano de atuação do MINUET. Em seguida, os componentes da arquitetura MINUET são detalhados e as suas interações, bem como é ilustrado o seu funcionamento.

\subsection{Modelo do Ambiente Urbano}

O ambiente urbano de atuação consiste de um espaço geográfico não estruturado formado por um conjunto de construções compartilhando espaço com malhas viárias onde veículos trafegam continuamente obedecendo legislações de tráfego, como ilustra a Figura 1. Neste ambiente, os veículos que trafegam são capazes de se comunicarem uns com os outros, Veículos-a-Veículos (V2V), e com a Infraestrutura Urbana através de Estações Bases (EB), Veículos-a-Infraestrutura (V2I), criando assim redes veiculares híbridas. Como ocorrem em zonas urbanas reais, eventos urbanos emergenciais de naturezas distintas podem surgir aleatoriamente no tempo e no espaço. Um evento urbano emergencial é qualquer evento que possa impactar no dia-a-dia da cidade e dos seus cidadãos, como por exemplo, incêndios, acidentes, delitos, obstruções nas vias, dentre outros. Estes eventos devem ser tratados por uma Entidade Externa (EE), representando uma autoridade competente, a qual se comunica, através das EBs, com a rede veicular não-estruturada.

$\mathrm{O}$ modelo de ambiente urbano assumido é composto por um conjunto $V$ de $n$ veículos (nós) identificados por $\left\{v_{1}, v_{2}, \ldots, v_{n}\right\}$ e um conjunto $E$ de $m$ eventos identificados por $\left\{e_{1}, e_{2}, \ldots, e_{m}\right\}$, onde qualquer veículo $v_{i} \in V$ pode detectar um evento $e_{j} \in E$, desde que $e_{j}$ esteja no raio de alcance de $v_{i}$. Esta detecção acontece por meio de dispositivos de geolocalização e/ou sensores embarcados, e assume-se por raio de alcance a distância máxima do evento ao veículo de forma que o dispositivo embarcado consiga detectar o evento. Todo evento possui um período de existência, que vai desde o instante de sua ocorrência até o seu natural sumiço ou tratamento final (definido pela EE), e a sua posição no espaço pode variar ao longo do tempo, caracterizando assim um evento fixo ou móvel. Uma vez detectado, o evento precisa ser monitorado ao longo do tempo por um grupo de veículos, sendo que a formação de um grupo depende do evento ser detectado. Portanto, denota-se por $E^{\prime} \subset E$, o conjunto de todos os eventos que foram detectados. Assim, para cada $e v \in E^{\prime}$, definimos um grupo de veículos de monitoramento $G m(e v)=\{v m\}$, onde $v m \subset V$. Então o modelo urbano ainda possui um conjunto $G$ de $r$ grupos de monitoramento identificados por $\left\{G m_{1}, G m_{2}, \ldots, G m_{r}\right\}$. Vale ressaltar que o tamanho do grupo associado a um evento é dinâmico, ou seja, ele pode variar com o passar do tempo devido à mobilidade dos veículos e do próprio evento. Os veículos podem entrar e sair do grupo, conforme a densidade de veículos nas vias e de suas direções.

Na Figura 1, as elipses em vermelho representam os grupos de veículos de monitoramento responsáveis pelo monitoramento e disseminação da informações dos eventos existentes em um dado instante de tempo. A mobilidade do evento deve ser levada em conta tendo em vista que os grupos devem se manter próximos dos seus respectivos eventos. Logo, pode-se concluir que um grupo responsável por monitorar um evento móvel deve acompanhá-lo o maior tempo possível, enquanto que um grupo responsável por mo- 


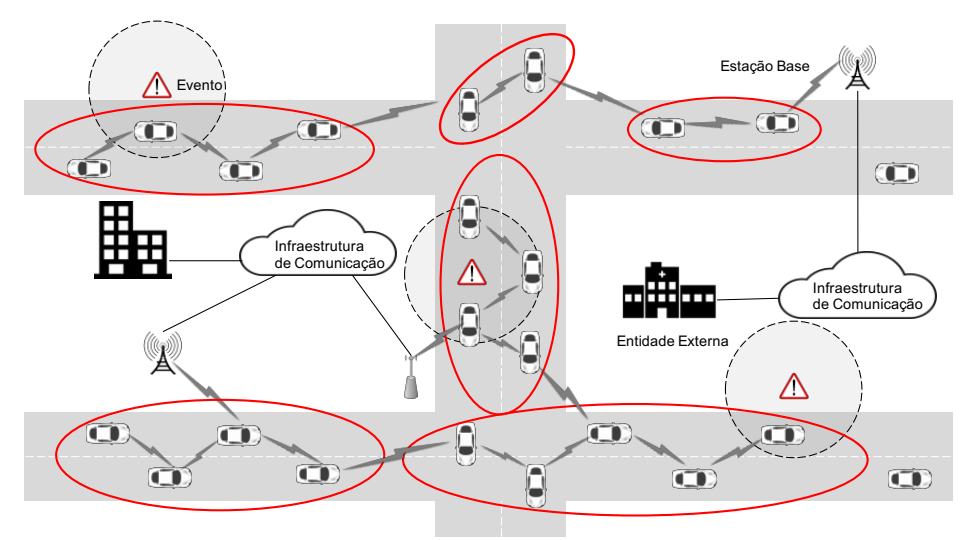

Figura 1. Modelo do ambiente urbano

nitorar um evento fixo tende a se afastar do respectivo evento (tomando o evento fixo como referencial). Assim, devido à alta dinamicidade da rede veicular e do eventos, intervalos de intermitência podem surgir resultando em possíveis lapsos no monitoramento.

Finalmente, para possibilitar um melhor tratamento do evento, os dados monitorados devem ser entregues à EE em um limite de tempo máximo de forma a serem passíveis de uso pelas suas respectivas aplicações alvo, e assim serem úteis à EE durante a tomada de decisão à resolução do evento. Obviamente, este limite de tempo depende diretamente da natureza do evento. Por exemplo, o limite de tempo para entregar os dados monitorados do evento obstrução na via pode ser maior do que aquele associado a um evento veículo em fuga. Define-se então o máximo de tempo de entrega $M T E(e v)$ como o intervalo de tempo máximo que o dado monitorado de $e v \in E^{\prime}$ deve ser entregue ao destino.

\subsection{MINUET}

Para garantir o monitoramento cooperativo e dinâmico de eventos apoiado por agrupamentos é proposto o sistema MINUET (MonitorINg and Dissemination of Urban EvenTs). A arquitetura do MINUET é composta por dois módulos principais, chamados Agrupamento e Controle, como ilustrado na Figura 2. O módulo Agrupamento configura a formação e a manutenção dos grupos de veículos que irão monitorar o evento. $\mathrm{O}$ módulo Controle é responsável pela coordenação da detecção, anúncio e monitoramento dos eventos e pela disseminação das informações destes eventos às EEs.

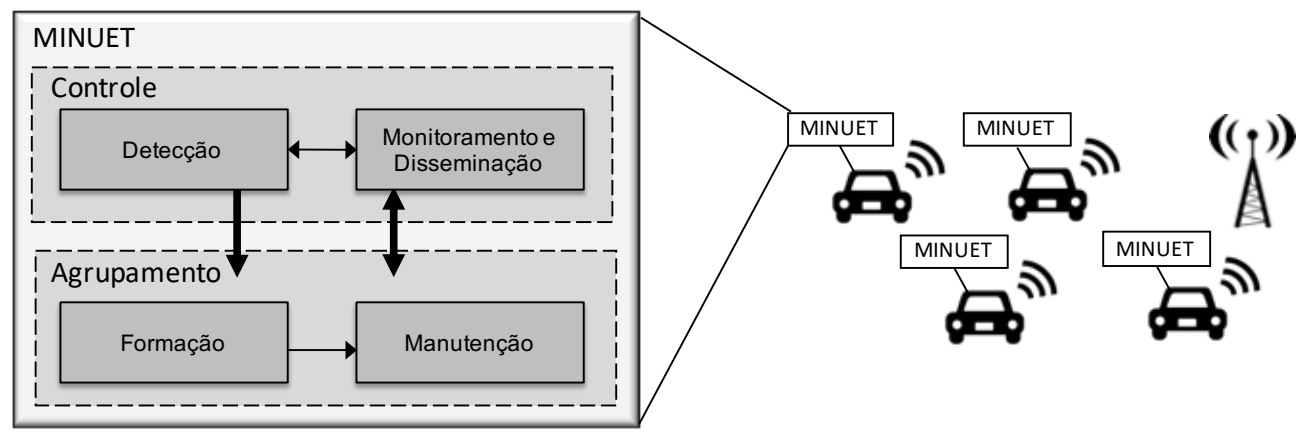

Figura 2. Arquitetura do MINUET

O módulo Agrupamento: tem por objetivo a criação e manutenção de grupos de veículos. Este módulo é composto por dois componentes: Formação e Manutenção. 
O componente Formação é responsável pela descoberta da vizinhança, seleção do líder do grupo, definição dos papéis de cada nó no grupo e estabelecimento da comunicação dos nós com seus respectivos líderes. Inicialmente, um nó deve anunciar periodicamente sua existência para seus vizinhos enquanto que, simultaneamente, obtém informações semelhantes dos mesmos com o objetivo de construir a topologia de rede. Com base nas informações dos vizinhos, nós podem fazer ou não parte de um grupo. Aqueles que são escolhidos para fazer parte do grupo são denominados de nós membros. Além disso, um nó líder é selecionado para exercer funções de manutenção do grupo.

Uma vez formado o grupo, o componente de Manutenção é o responsável pelo reagrupamento e/ou refiliação de membros no grupo. Considerando fatores como a alta velocidade dos nós, mobilidade do evento, variação topológica da rede, comportamento dos condutores e interferências no canal, a frequência de entrada e saída de nós nos grupos pode variar consideravelmente com o tempo. Por isso é necessária a criação de mecanismos que consigam lidar com esta alta dinamicidade da rede sem comprometer o seu desempenho. Este componente também trata da sobreposição de grupos, quando dois ou mais grupos compartilham uma determinada região em um mesmo instante de tempo. Nestes casos, pode haver a necessidade de mesclar estes grupos sobrepostos em apenas um só. Salienta-se que diferentes técnicas de agrupamento podem ser usadas, variando assim os seus mecanismos de formação e manutenção de grupos, conforme cada caso.

O módulo Controle: é responsável por coordenar a detecção, o monitoramento do evento no grupo formado e a disseminação das informações para a EE. Ele é formado por dois componentes, sendo o primeiro responsável por detectar e anunciar o evento e o segundo componente por monitorar e disseminar as informações do evento detectado. Para isso, além da gestão do grupo em si, é necessário a gestão e o controle de monitoramento no grupo, onde veículos específicos devem se auto-escalonar dinamicamente para colaborar no tratamento de múltiplos eventos sobre diferentes tipos de cenários de tráfego. Assim, além dos papéis definidos pelo módulo de Agrupamento, este módulo também estabelece outros três papéis, que são: (i) Monitorador: enquanto um evento estiver no raio de alcance de um veículo, o mesmo será capaz de monitorá-lo. (ii) Retransmissor: veículo que apenas dissemina as informações dentro do grupo com o intuito de alcançar a EE. (iii) Gateway: veículo que entrega as informações do evento monitorado à EB e, consequentemente, à EE. O gateway também é o responsável por repassar as informações entre grupos. Um veículo também pode exercer mais de um papel simultaneamente. Por exemplo, um veículo pode ser tanto monitorador, porque está próximo do evento, como gateway, porque está ao alcance da EB.

O componente Detecção trata inicialmente da análise e detecção de um evento por um veículo. Um veículo $v d$ na via de trânsito, pode detectar a ocorrência de um evento $e v$ em um determinado instante de tempo. Após a detecção, $v d$ coleta as dados de contexto de $e v$, que são instante de tempo da detecção, localização, velocidade e direção. Se o evento é fixo, então sua velocidade é zero. Por razões de simplificação, assumimos que a análise e detecção do evento são realizadas através de técnicas de processamento e análise de imagens obtidas por sensores de câmeras embarcadas nos veículos. Além disso, assumimos também que durante a análise do evento, $v d$ consegue estimar o $M T E(e v)$.

Após a etapa de coleta das informações de contexto, inicia-se o processo para disseminá-las, através de mensagens de anúncio ao longo da rede. Contudo, nem todos 
os veículos da malha viária urbana necessitam ter ciência da ocorrência do evento. Além disso, após um determinado tempo, o anúncio de um evento não é mais útil. Desta forma, pretende-se limitar a disseminação do anúncio a um determinado intervalo de tempo, reduzindo assim o overhead e possíveis perdas e atrasos na rede. Considerando que um veículo $v r$ receba a mensagem de anúncio no instante $t r$ e que o evento foi detectado no instante $t d$, então $v r$ repassa a mensagem se o tempo que ainda tem para retransmitíla ainda não ultrapassou o limite de tempo de entrega referente ao evento $(t r-t d \leq$ $M T E(e v))$. Caso contrário, a mensagem é descartada. Este limite temporal calculado por cada veículo que recebe a mensagem de anúncio delimita uma área chamada de Zona de Anúncio (ZA), a qual determina os veículos que podem fazer parte do grupo para colaborar no monitoramento do evento.

A Figura 3 ilustra um evento detectado e a ZA gerada com base neste evento, bem como os veículos que fazem parte da ZA. O evento é detectado pelos veículos VDl e $V D 2$, e as mensagens de anúncio seguem as rotas estabelecidas pelas setas. Percebe-se que apesar do veículo $A$ estar geograficamente mais perto do evento do que o veículo $B(d A<d B), B$ faz parte da ZA, enquanto que $A$ não. Isto pode acontecer quando a mensagem de anúncio demorar mais para chegar em $A$ do que em $B$, o que pode fazer com que o $M T E$ seja ultrapassado em $A$. Na Figura 3, a rota que a mensagem segue para chegar em $A$ possui mais saltos do que a rota para $B$, ocasionando assim o atraso e o descarte da mensagem em $A$. Todos os outros veículos continuam disseminando a mensagem. Após um veículo receber uma mensagem de anúncio e confirmar que está na ZA, ele então verifica se fará parte do grupo que colaborará ao monitoramento do evento. Esta verificação é feita pelo módulo Agrupamento, de acordo com a técnica e o serviço de agrupamento desenvolvido e utilizado para apoiar a disseminação de informação.

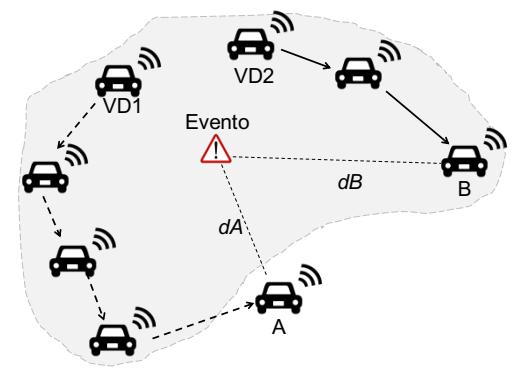

Figura 3. Zona de anúncio

Por fim, o componente de Monitoramento e Disseminação trata do monitoramento do evento e da disseminação de seus dados pelo grupo até entregá-las a uma EB. Para isto, este componente cuida do auto-escalonamento de papéis entre os veículos para o monitoramento cooperativo. Quando o veículo $v d$ detecta o evento $e v$, ao mesmo tempo que realiza a disseminação do anúncio, também inicia o monitoramento de $e v$, coletando seus dados e disseminando através de mensagens de monitoramento. O veículo $v d$ será então um monitorador enquanto $e v$ estiver no seu raio de alcance. Caso $v d$ também estiver ao alcance de uma EB, os dados coletados são entregues, fazendo com que $v d$ também seja um gateway. Caso contrário, $v d$ apenas dissemina as mensagens para cada vizinho. Cada veículo que recebe a mensagem de monitoramento, primeiro verifica se faz parte do grupo formado. Caso faça parte, também dissemina as mensagens, assumindo o papel de retransmissor. Se o veículo estiver ao alcance de uma EB, então ele assume o papel de 
gateway, entregando assim as mensagens de monitoramento.

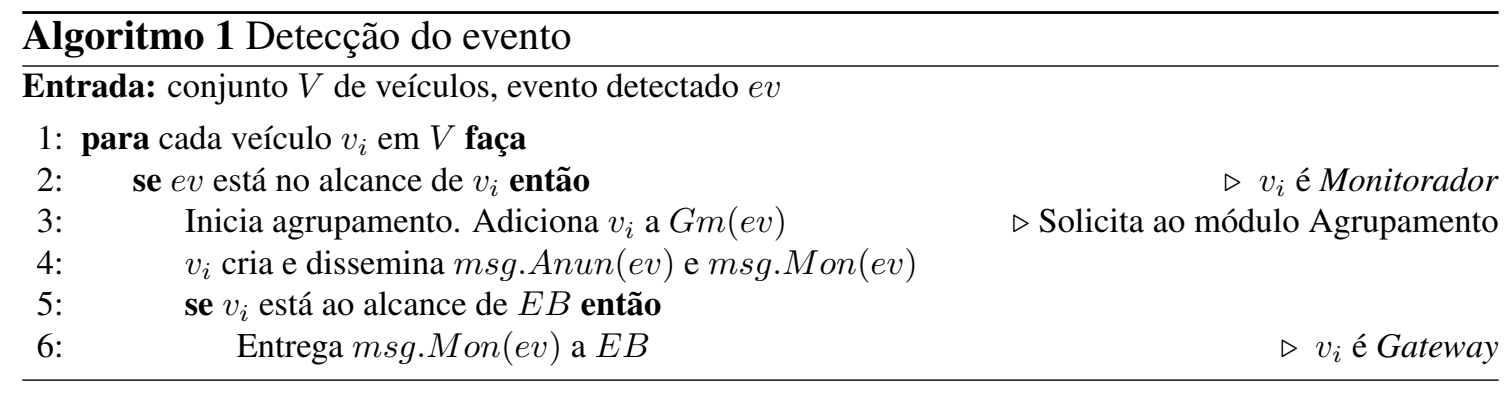

O Algoritmo 1 descreve a operação que ocorre nos veículos que conseguem detectar o evento $e v$. Uma vez que $e v$ é detectado, o veículo $v_{i}$ assume o papel de monitorador e inicia a formação do grupo, que é realizado pelo módulo Agrupamento. Em seguida, $v_{i}$ cria e dissemina as mensagens de anúncio e monitoramento. A mensagem de anúncio (msg.Anun $(\mathrm{ev})$ ) contém as informações de contexto do evento, necessárias para a manutenção do grupo formado, como por exemplo, a posição do evento, o tempo de detecção e o tipo do evento. A mensagem de monitoramento (msg.Mon(ev)) contém os dados monitorados do evento. Além disso, se $v_{i}$ estiver ao alcance de uma EB, também assume o papel de gateway e entrega $m s g . \operatorname{Mon}(e v)$ à EB.

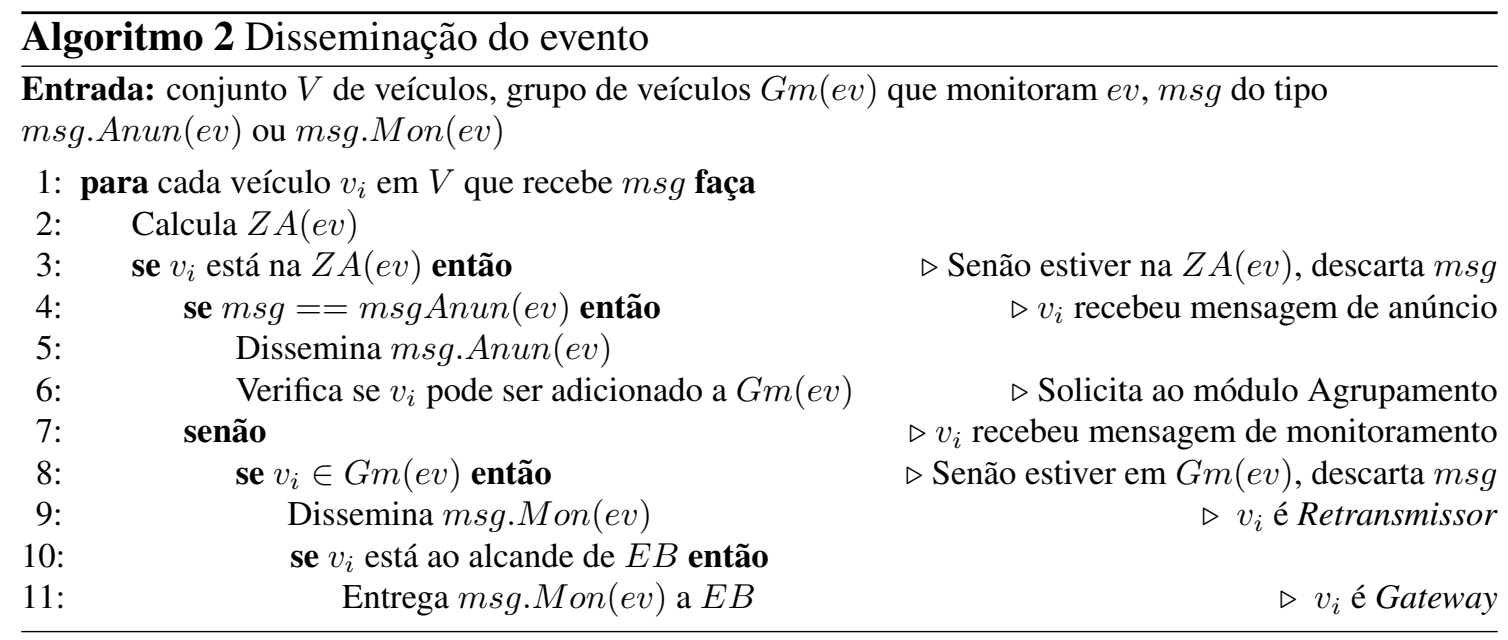

Já nos veículos que não detectaram o evento e receberam uma mensagem de anúncio ou monitoramento, é necessário verificar se eles devem fazer parte do grupo de monitoramento e disseminar tais mensagens (Algoritmo 2). Toda vez que um veículo $v_{i}$ recebe uma mensagem, ele deve verificar se está na $Z A$ de $e v$. Se não estiver, descarta a mensagem. Caso esteja, se for uma mensagem de anúncio, ele dissemina a mensagem e verifica (através do módulo Agrupamento) se pode fazer parte do grupo $G m(e v)$ que está monitorando $e v$. Se $v_{i}$ receber uma mensagem de monitoramento, então só é possível disseminar se já fizer parte de $G m(e v)$, fazendo neste caso que $v_{i}$ seja um retransmissor. Além disso, se $v_{i}$ estiver ao alcance de uma $E B$, também faz o papel de gateway e entrega a mensagem à $E B$. Caso não faça parte do grupo, descarta a mensagem.

A Figura 4 exemplifica um cenário de operação do MINUET em um intervalo de tempo $t$ contendo o conjunto $V=\{A, B, C, D, E, F, G, H\}$ e o conjunto $E=\{E V\}$. Os veículos $D$ e $E$ conseguem detectar o evento $E V$, criando assim o conjunto $E^{\prime}=\{E V\}$. 


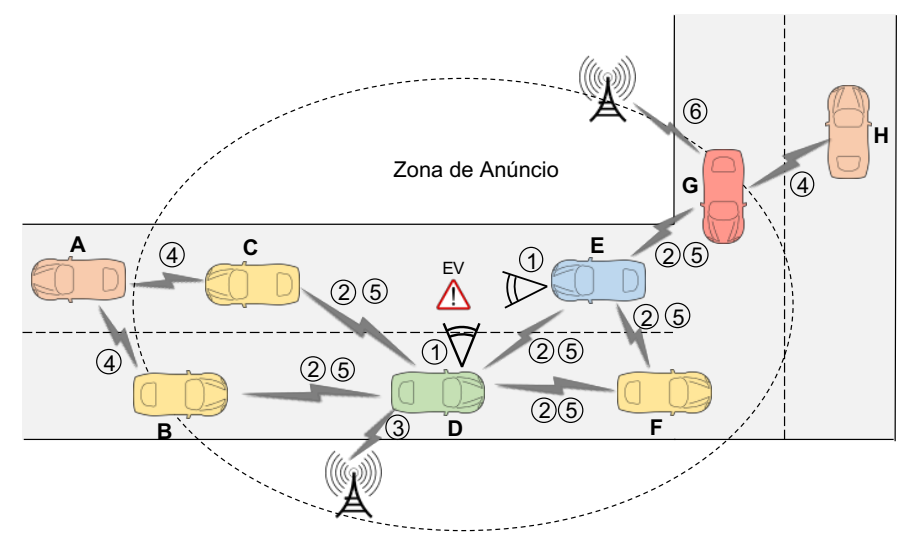

Figura 4. Exemplo de operação do MINUET

$D$ e $E$ coletam então os dados de contexto de $E V$ (1), assumindo o papel de monitoradores. Imediatamente iniciam a disseminação das mensagens de anúncio (2). O veículo $D$, por estar ao alcance de uma EB, também entrega os dados monitorados (3), tornando-se assim também um gateway. Conforme as mensagens de anúncio vão sendo disseminadas, os veículos verificam se fazem parte da ZA e do grupo formado para realizar o monitoramento cooperativo. Os veículos $A$ e $H$, ao receberem a mensagem de anúncio (4), verificam que não fazem parte da ZA, não participando assim do monitoramento de $E V$. Para efeitos de simplificação, consideramos que todos os veículos que estão na ZA também fazem parte do grupo. Assim, é criado então o grupo $G m(E V)=\{B, C, D, E, F, G\}$. À medida que o grupo vai sendo formado, as mensagens de monitoramento também vão sendo disseminadas através dos seus membros (5). Os veículos $B, C$ e $F$ assumem então o papel de retransmissores, enquanto o veículo $G$, por conseguir entregar a mensagem de monitoramento à uma EB (6), assume o de gateway.

\section{Avaliação}

Esta seção apresenta uma avaliação do sistema MINUET para verificar a eficácia e eficiência no monitoramento e disseminação de eventos emergenciais urbanos. O MINUET foi implementado em C++ e testado no simulador NS3 juntamente com o SUMO. Foi utilizado um recorte do LuST (Luxembourg SUMO Traffic) como cenário de mobilidade de tráfego, onde os padrões de tráfego seguem os dados reais da cidade de Luxemburgo. O algoritmo empregado para realizar o agrupamento foi o DCA [Tal et al. 2016], ressaltando-se que outras técnicas de agrupamento podem ser utilizadas. Foram realizadas simulações em dois cenários distintos, ambos com um evento fixo e considerando diferentes velocidades média dos veículos, número de faixas na via de trânsito, sentido da via e distância da EB até o evento. Os dois cenários foram usados para avaliar como estas variações afetam no desempenho do MINUET. Em ambos os cenários ocorre apenas um evento com duração total de 10 mins.

A Figura 5 ilustra as vias onde ocorre a simulação, sendo que o centro das elipses indicam a posição onde ocorre o evento no cenário $\mathrm{C} 1$ e $\mathrm{C} 2$. Veículos que participam do grupo de monitoramento podem trafegar tanto na via onde o evento está localizado como em vias próximas. A Tabela 1 sumariza os parâmetros utilizados nas simulações dos dois cenários. As métricas utilizadas para aferir a eficácia e eficiência de MINUET foram: Número de veículos agrupados $(N v a)$, Número de mensagens de monitoramento 
entregues ( $N m e$ ), Tempo de notificação do evento (Tne), Taxa de mensagens de monitoramento entregues ( $T$ me), Taxa de mensagens de monitoramento entregues duplicadas $(T m d)$ e Número de detecções $(N d)$. A Tabela 2 descreve as métricas avaliadas.

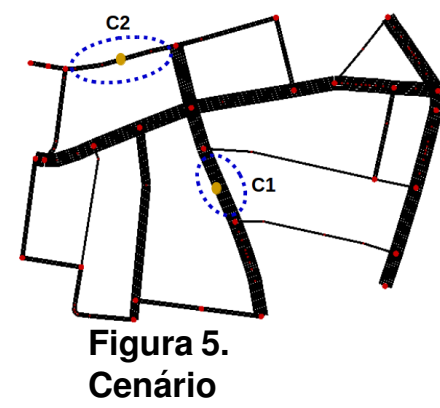

Tabela 1. Configurações das simulações

\begin{tabular}{lll}
\hline Parâmetro & C1 & C2 \\
\hline Tempo da simulação & $12 \mathrm{mins}$ & $12 \mathrm{mins}$ \\
Número de Eventos & 1 & 1 \\
Tempo de duração do evento & $10 \mathrm{mins}$ & $10 \mathrm{mins}$ \\
Velocidade média dos veículos & $10,69 \mathrm{~m} / \mathrm{s}$ & $14,38 \mathrm{~m} / \mathrm{s}$ \\
Sentido da via & mão dupla & mão única \\
Número de faixas & 6 & 2 \\
Distância do evento até a EB & $130 \mathrm{~m}$ & $160 \mathrm{~m}$ \\
Alcance de transmissão veículos & $100 \mathrm{~m}$ & $100 \mathrm{~m}$ \\
\hline
\end{tabular}

Tabela 2. Métricas aplicadas na avaliação

\begin{tabular}{ll}
\hline Métrica & Descrição \\
\hline Nva & $\begin{array}{l}\text { corresponde ao número de veículos participantes de um grupo } \\
\text { corresponde ao número total de mensagens de monitoramento que foram entregues a }\end{array}$ \\
Tne & $\begin{array}{l}\text { EB } \\
\text { corresponde ao intervalo de tempo entre o instante que o evento é detectado a primeira } \\
\text { vez e o instante que a primeira mensagem de monitoramento é entregue à EB (Te) } \\
\text { corresponde ao percentual de mensagens de monitoramento enviadas pelos monitora- } \\
\text { dores que foram entregues à EB } \\
\text { corresponde ao percentual de mensagens de monitoramento entregues a EB que são } \\
\text { réplicas }\end{array}$ \\
$N d$ & corresponde ao número de vezes que o evento foi detectado durante a sua ocorrência \\
\hline
\end{tabular}

\subsection{Resultados}

A Figura 6 apresenta o desempenho do MINUET referente à detecção do evento e à entrega dos dados monitorados à EB. O gráfico 6(a) mostra o número de veículos por grupo ( Nva ) ao longo do tempo nos dois cenários, sendo que um grupo é formado a cada vez que o evento é detectado (Evento Detectado - ED). É possível perceber tanto momentos de intermitência do monitoramento, quanto momentos em que grupos formados mudam de tamanho ao longo do tempo, como destacados pelas elipses. Estas mudanças de tamanho são o resultado direto das funções de gerência do agrupamento, que controlam quando veículos entram e deixam o mesmo grupo. Isto demonstra o monitoramento cooperativo dos veículos alcançado pelo MINUET durante o monitoramento do evento (crowdsourcing). O gráfico também mostra que no cenário de $14 \mathrm{~m} / \mathrm{s}$, o número de veículos por grupo é maior que no cenário de $10,14 \mathrm{~m} / \mathrm{s}$. Isto se deve ao fato da densidade da via ser maior, logo há mais veículos trafegando. Também é possível notar que há uma grande quantidade de grupos com apenas um veículo. Isto é explicado porque o veículo monitorador está isolado, ou seja, não há nenhum outro veículo próximo o suficiente para participar do seu grupo de forma a entregar os dados monitorados a EB.

O gráfico 6(b) mostra a eficácia do MINUET ao entregar as mensagens de monitoramento à EB (Evento Recebido - ER). É possível observar que nem sempre que o evento é detectado, as mensagens de monitoramento são entregues. Isto acontece quando não há veículo algum do grupo próximo o suficiente da EB para entregar as mensagens. Além disso, ao comparar com o gráfico 6(a), as mensagens são entregues quando o Nva 
é maior, o que também faz sentido, uma vez que a probabilidade de haver um veículo ao alcance da EB é maior. Isto também explica o fato de haver mais mensagens entregues no cenário 2, já que o seu $N v a$ médio é maior do que o $N v a$ do cenário 1. Também pode-se observar que há mais mensagens sendo entregues (destacadas pelas elipses no gráfico) quando os grupos passam mais tempo formados. Ou seja, é possível inferir que a coordenação e a colaboração dentro do grupo formado é que permitem a entrega das mensagens a EB, demonstrando assim o controle distribuído alcançado pelo MINUET.

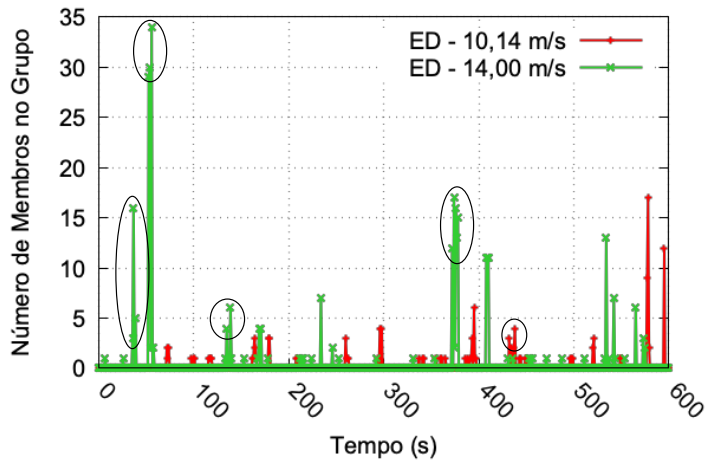

(a) Grupos formados

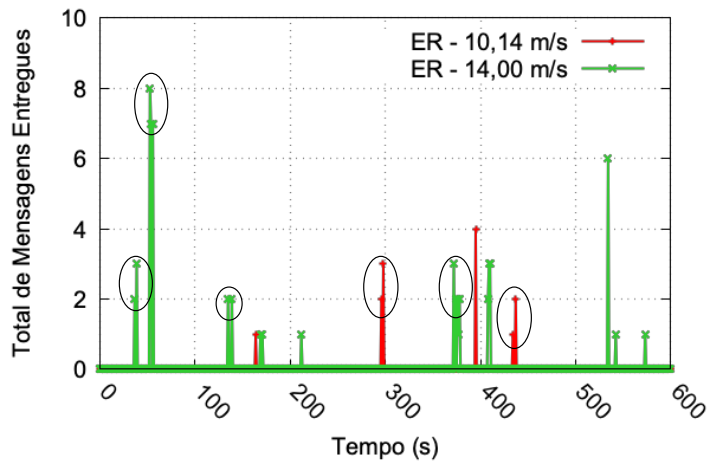

(b) Mensagens entregues

Figura 6. Detecção do evento e entrega das mensagens de monitoramento

Os gráficos da Figura 7 apresentam o custo computacional do MINUET no monitoramento cooperativo. O gráfico 7(a) mostra o tempo decorrido entre o instante da primeira vez que o evento é detectado e o instante de notificação do evento (Tne), o que corresponde à primeira mensagem de monitoramento que chega na EB. Apesar de que no cenário 2 a velocidade média dos veículos é maior, o seu Tne é menor. Isto é explicado pelo fato de que no cenário 2 é formado um grupo com um $N v a$ maior e mais cedo do que no cenário 1, possibilitando assim a entrega da mensagem de monitoramento. Já o gráfico 7(b) apresenta o número de vezes que o evento foi detectado ao longo de sua ocorrência $(N d)$. No cenário 1 o evento foi detectado mais de 60 vezes enquanto que no cenário 2 foi detectado cerca de 55 vezes. Apesar da intermitência de detecção, o sistema MINUET conseguiu alcançar uma média de 6 detecções por minuto.

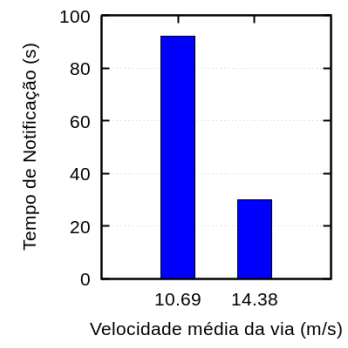

(a) Tne

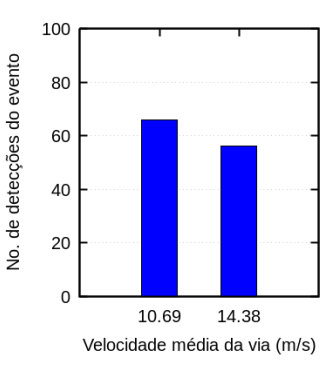

(b) $\mathrm{Nd}$

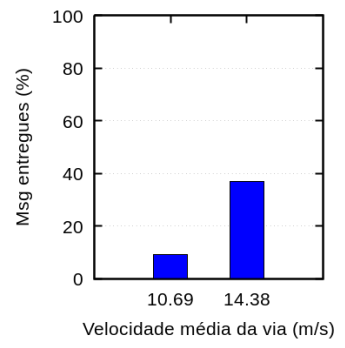

(c) Tme

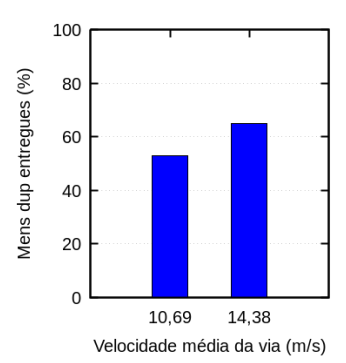

(d) $T m d$

Figura 7. Notificação evento, msgs entregues, msgs duplicadas e no. detecções

O gráfico 7(c) apresenta a percentual de mensagens de monitoramento não duplicadas entregues a EB (Tme). É possível ver que no cenário 2, o Tme chega perto de $40 \%$, o que garante uma taxa razoável de dados coletados do evento, considerando o ambiente dinâmico onde ocorre. Finalmente, o gráfico 7(d) apresenta o percentual de réplicas de 
mensagens de monitoramento entregues a EB $(T m d)$. Neste caso, os resultados mostram que nos dois cenários, mais de $50 \%$ das mensagens entregues são réplicas. Isto acontece pois pode haver mais de um gateway ao mesmo tempo em um grupo formado. Vale ressaltar que os resultados foram alcançados ao utilizar o algoritmo DCA. Extensões do trabalho estão em andamento utilizando outras técnicas de agrupamento, eventos móveis e sobrepostos no tempo e espaço para averiguar a escalabilidade da proposta.

\section{Conclusões}

Este trabalho apresentou o sistema MINUET para apoio ao monitoramento cooperativo de eventos emergenciais urbanos através de agrupamento sob demanda de veículos. O MINUET consegue tratar eventos aleatórios no tempo e espaço em um ambiente urbano através do monitoramento e disseminação cooperativos dos dados coletados até uma entidade externa. Uma vez na entidade externa, estes dados podem ser interpretados, gerando informações para auxiliar a entidade na tomada de decisão referente à resolução do evento. Resultados de simulações mostraram que o MINUET consegue detectar um evento, formar grupos que colaboram para a disseminação de mensagens de monitoramento e entregar as mensagens a uma EB através destes grupos. Estas mensagens podem ser usadas pela EE para auxiliar no processo de tomada de decisão referente à resolução do evento. Como trabalhos futuros pretende-se analisar o desempenho do MINUET em cenários com eventos sobrepostos no tempo e espaço, além de considerar eventos móveis e utilizar outras técnicas de agrupamento.

\section{Referências}

Abhishek, R., Zhao, S., and Medhi, D. (2016). Spartacus: Service priority adaptiveness for emergency traffic in smart cities using software-defined networking. In 2016 IEEE International Smart Cities Conference (ISC2), pages 1-4.

Castro, J., Araujo, I., Anjos, E., and Matos, F. (2017). A Survey on Bus Monitoring Systems. In Computational Science and Its Applications - ICCSA 2017, Lecture Notes in Computer Science, pages 220-231.

Chou, Y., Chu, T., Kuo, S., and Chen, C. (2017). An Adaptive Emergency Broadcast Strategy for Vehicular Ad Hoc Networks. IEEE Sensors Journal, 18(12):4814-4821.

Furutai, K. and Kanno, T. (2018). Resilience Analysis of Urban Critical Infrastructure: A human-Centred View of Resilience. Technical report, EPFL International Risk Governance Center.

Gorrieri, A., Martalò, M., Busanelli, S., and Ferrari, G. (2016). Clustering and sensing with decentralized detection in vehicular ad hoc networks. Ad Hoc Networks, 36:450 - 464. Vehicular Networking for Mobile Crowd Sensing.

Hassan, A. M. and Awad, A. I. (2018). Urban transition in the era of the internet of things: Social implications and privacy challenges. IEEE Access, 6:36428-36440.

Huang, C., Wang, H., Zhou, H., Xu, S., and Ren, D. (2017). EVAC-AV: The Live Road Surveillance Control Scheme Using an Effective-Vision-Area-Based Clustering Algorithm With the Adaptive Video-Streaming Technique. IEEE Systems Journal, 11(3):1228-1238.

Khakpour, S., Pazzi, R. W., and El-Khatib, K. (2013). A Distributed Clustering Algorithm for Target Tracking in Vehicular Ad-hoc Networks. In Proceedings of the Third ACM 
International Symposium on Design and Analysis of Intelligent Vehicular Networks and Applications, DIVANet '13, pages 145-152, New York, NY, USA. ACM.

Khakpour, S., Pazzi, R. W., and El-Khatib, K. (2017). Using clustering for target tracking in vehicular ad hoc networks. Vehicular Communications, 9:83-96.

Ma, F., Liu, X., Liu, A., Zhao, M., Huang, C., and Wang, T. (2018). A Time and Location Correlation Incentive Scheme for Deep Data Gathering in Crowdsourcing Networks. Wireless Communications and Mobile Computing, 2018:22.

Macedo, R., Melo, R., Santos, A., and Nogueira, M. (2014). Experimental performance comparison of single-path and multipath routing in VANETs. In GIIS, pages 1-6.

Memos, V. A., Psannis, K. E., Ishibashi, Y., Kim, B.-G., and Gupta, B. (2018). An efficient algorithm for media-based surveillance system (eamsus) in iot smart city framework. Future Generation Computer Systems, 83:619 - 628.

Monajemi, S., Sanei, S., and Ong, S.-H. (2018). Information reliability in complex multitask networks. Future Generation Computer Systems, 83:485 - 495.

Monreal, C. O. (2018). Future urban mobility group: Smart cities research institute swinburne university of technology, melbourne australia [its research lab]. IEEE Intelligent Transportation Systems Magazine, 10(2):203-205.

Mousa, M., Zhang, X., and Claudel, C. (2016). Flash flood detection in urban cities using ultrasonic and infrared sensors. IEEE Sensors Journal, 16(19):7204-7216.

Quadros, C., Cerqueira, E., Santos, A. L. d., Lim, J.-H., and Gerla, M. (2015a). Beaconless video streaming management for VANETs based on QoE and link-quality. In IEEE IM, pages 191-198.

Quadros, C., Santos, A., Gerla, M., and Cerqueira, E. (2016). QoE-driven dissemination of real-time videos over vehicular networks. Comp. Communications, 91-92:133-147.

Quadros, C., Santos, A. L. d., Gerla, M., and Cerqueira, E. (2015b). A QoE-Aware Mechanism to Improve the Dissemination of Live Videos over VANETs. In $S B R C$, pages 31-40. IEEE Computer Society.

Shi, C., Zhou, Y., Li, W., Li, H., Lu, N., Cheng, N., and Yang, T. (2017). A centralized clustering based hybrid vehicular networking architecture for safety data delivery. In GLOBECOM 2017 - 2017 IEEE Global Communications Conference, pages 1-6.

Silva, C., Nogueira, M., Kim, D., Cerqueira, E., and Santos, A. (2016). Cognitive radio based connectivity management for resilient end-to-end communications in VANETs. Computer Communications, 79:1-8.

Tal, I., Kelly, P., and Muntean, G. (2016). A novel direction-based clustering algorithm for vanets. In 23rd International Conference on Telecommunications (ICT), pages 1-5.

Timashev, S. A. (2017). Resilient urban infrastructures - basics of smart sustainable cities. IOP Conference Series: Materials Science and Engineering, 262(1):012197.

Wang, X. (2018). Data Acquisition in Vehicular Ad Hoc Networks. Commun. ACM, 61(5):83-88.

Wang, X., Ning, Z., Hu, X., Wang, L., Hu, B., Cheng, J., and Leung, V. C. M. (2018). Optimizing content dissemination for real-time traffic management in large-scale internet of vehicle systems. IEEE Transactions on Vehicular Technology, pages 1-1.

Yedder, H. B. and Benyahia, I. (2017). Reactive emergency vehicles dispatching based real-time information dissemination. In 2017 8th IEEE Annual Information Technology, Electronics and Mobile Communication Conference (IEMCON), pages 471-477. 